\title{
A CASE REPORT OF ACUTE NONTRAUMATIC SPONTANEOUS SUBDURAL HAEMATOMA DUE TO PSEUDOANEURYSM OF MIDDLE MENINGEAL ARTERY
}

\author{
Feda Makkiyah $^{1}$, Rahmah Nida Nurrahmah $^{2}$ \\ Correspondence: fedaanisah@upnvj.ac.id \\ ${ }^{1}$ Faculty of Medicine Universitas Pembangunan Nasional Veteran Jakarta, Indonesia \\ ${ }^{2}$ Faculty of Health Sciences Universitas Pembangunan Nasional Veteran Jakarta, Indonesia
}

\author{
Article History: \\ Received: Mei 18, 2020 \\ Accepted: December 1, 2020 \\ Published: Januaru 1, 2021

\section{Cite this as:} \\ Makkiyah $F$, Nurrahmah RN. A \\ case report of acute nontraumatic \\ spontaneous subdural \\ haematoma due to \\ pseudoaneurysm of middle \\ meningeal artery. Malang \\ Neurology Journal; 2021.7:66- \\ 69. \\ http://dx.doi.org/10.21776/ub.mnj \\ 2021.007.01.13
}

\section{ABSTRACT}

Acute subdural bleeding is blood collection in subdural space, usually caused by laceration of the bridging vein that runs from cortex to meningeal layer. On the other hand, the acute epidural hematoma is a collection of blood above the dura mater, usually caused by tearing of the middle meningeal artery. Subdural hematoma acute more likely happen in elderly than young adult. This case report discusses acute spontaneous subdural hematoma that was caused by a pseudoaneurysm of the middle meningeal artery. Seventeen years old boy came with symptoms of high intracranial pressure because of massive subdural bleeding. He was undergone craniotomy. After the clot removal operation, Digital Subtraction Angiography revealed vascular blush and pseudoaneurysm of the middle meningeal artery. Onyx embolization was delivered to proximal to a distal branch of the middle meningeal artery. The patient recovered in good condition, and no rebleeding in two years. Conclusion. Acute spontaneous subdural bleeding even though it is quite rare, but the chance of fatal rebleeding is high. As a result, one still has to look for the source of bleeding and manage comprehensively with embolization.

Keywords: Subdural hematoma, spontaneous, middle meningeal artery

\section{Introduction}

Subdural hematoma is bleeding in subdural space and mostly caused by trauma when it is in acute phase occur. This hematoma caused by laceration of bridging veins, which drain the venous blood from the cortex to the (SSS). This bridging veins bridge the subdural space. ${ }^{1}$ On the other hand, acute non traumatic or spontaneous subdural hematoma is a rare entity. 4 cases reported of cortical aneurysms ruptured. ${ }^{2,3}$ Spontaneous subdural hematoma happen quite frequently in elderly patients in chronic setting, because of brain atrophy caused the stretching of bridging veins and are prone to rupture regardless minimal trauma. However, the occurrence of acute spontaneous subdural hematoma is uncommon in young people. Here we reported the unusual spontaneous acute subdural hematoma in 17 years old patients. He came with severe progressive headache without any coagulopathy or any bleeding disorders. The suspicion of the source of bleeding was coming from malformation of arteries or vessels. The reason behind was because it was spontaneous non traum aand acute occurence.

Ruptured pseudoaneurysm of middle meningeal artery (MMA) which is run over the duramater is unlikely caused subdural hematoma. The laseration of middle meningeal artery which is most often trauma in origin usually collects in extradural space. This presented case discusses ruptured of MMA which is unlikely occured in subdural space.

\section{Case Report}

17 years old male patients were brought to emergency unit with the sudden, progressive headache when he was studying at the night before the next day examination. He had no history of trauma and denied taken any anticoagulants or have any previous illness. He vomit three times in emergency unit but still retained his consciousness. Physical examination: $\mathrm{BP}=130 / 80 \mathrm{mmHg} H R=80 \mathrm{x} / \mathrm{m}$, $\mathrm{RR}=12 \mathrm{x} / \mathrm{m}, \mathrm{T}=$ normal temperature. Neurological examination: $\mathrm{GCS}=14$, pupils were both reactive, equal and no paresis. Laboratory value was within normal limit. CT scan showed acute subdural hematoma in left frontoparietal with significant mass effect (Figure 1). Pasien was undergone craniotomy procedure to remove the blood clot and duramater was closed water-tight. Post operative scan shows that the subdural hematoma was minimal and no midline deviation. (Figure 2).

DSA was done the next day to find out source of bleeding. Left internal carotid artery ( L ICA) flushing showed normal branches (Figure 3). When the contrast agent was injected to the left external carotid artery ( L ECA), it was seen that the ipsilateral middle meningeal artery had abnormal size and there was vascular blush in the distal branch of middle meingeal artery and duramater was hyperemia. (Figure 4).

After neuroendovascular team discussion was held in a few minutes after DSA, he was suggested to undergo 
embolization with Onyx. He was agreed with the suggested treatment of embolization. Excelsior SL 10 microcatheter (Boston USA) was inserted in the proximal part of middle meningeal artery. After confirming the presence of collateral vascularization between ophtalmica artery and middle meningeal artery, then onyx was inserted. The result of onyx embolization was returning size of middle meningeal artery and absence of vacular blush. (Figure 5). Patient was discharge without any neurological deficits and had no recurrence in the next two years.

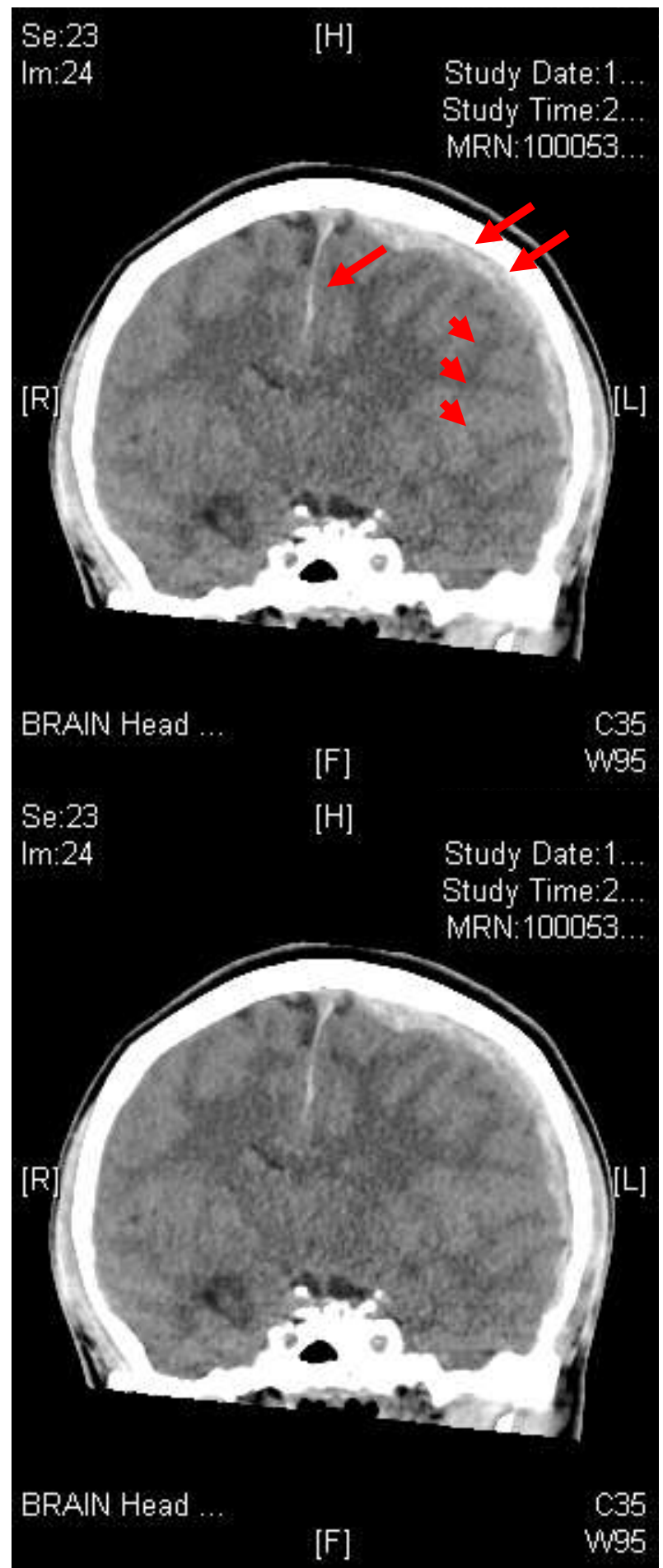

Figure 1. Coronal head CT scan shows left acute subdural hematoma (two red arrows) with significant deviation of midline (one red arrow), left lateral ventricle (frontal horn) is not visible. There is effacement of left sulci and gyri (three small red arrows).

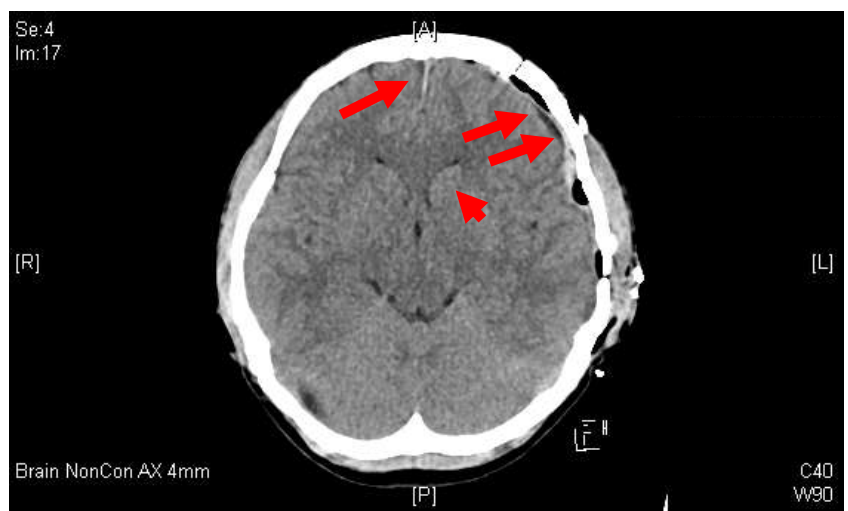

Figure 2. 6 hours after craniotomy head axial CT scan. It shows less subdural hematoma (two red arrows) and no deviation of midline (one red arrow). Sulci and gyri are opened. Left frontal horn of lateral ventricle is visible (a small red arrow).

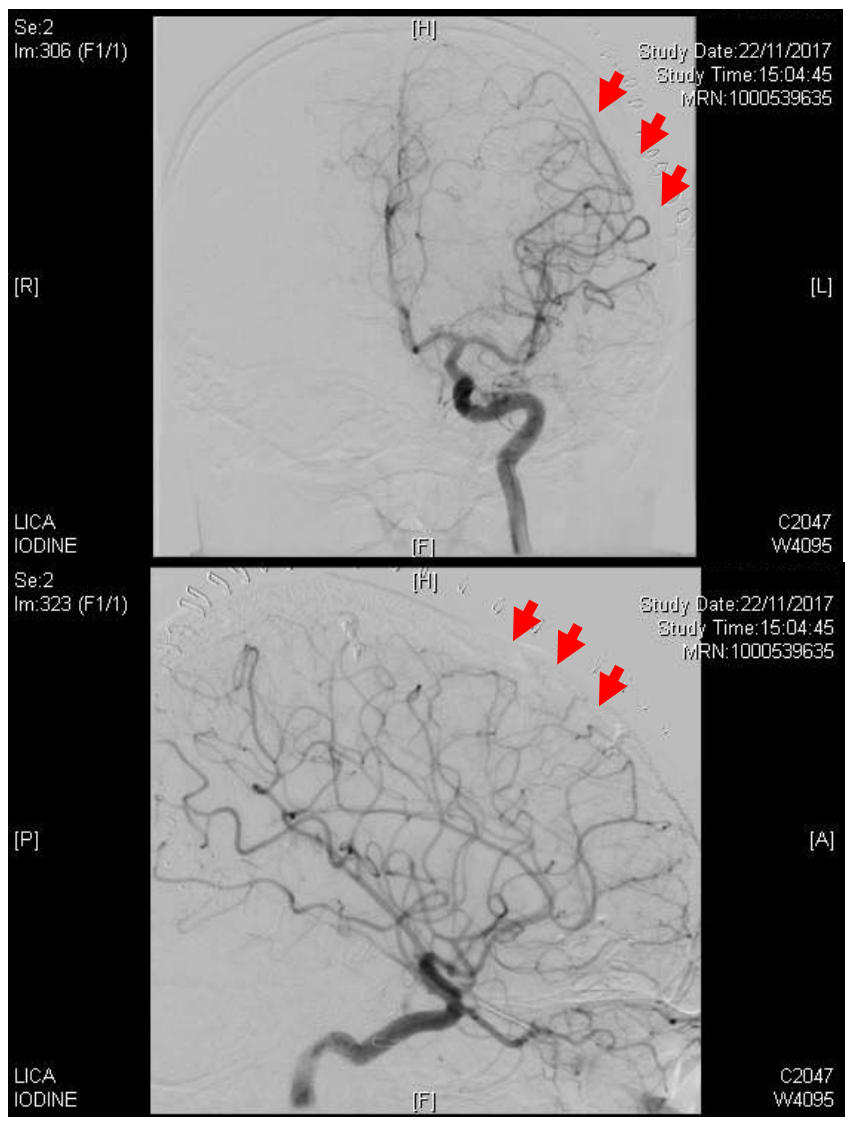

Figure 3. Digital substration angiography of Left Internal Carotid Artery shows no vascular abnormalities (three red arrows).

\section{Discussion}

Subdural hematoma acute is an urgent neurosurgical situation that might cause death and most of the cases are trauma in origin. ${ }^{4}$ Nonetheless, acute nontraumatic origins are such as ruptured of intracranial aneurysms, ruptured cortical arteries, hypertension, hematologic diseases, anticoagulant use and thromobolysis, cerebral amyloid angiopathy (CAA), dural arteriovenous fistules and acquired immune deficiency syndrome. ${ }^{2,4}$

A case of acute subdural hematoma that was caused of pseudoaneurysm of middle meningeal artery was reported in 77 years old female patients that consumed enoxiparin 


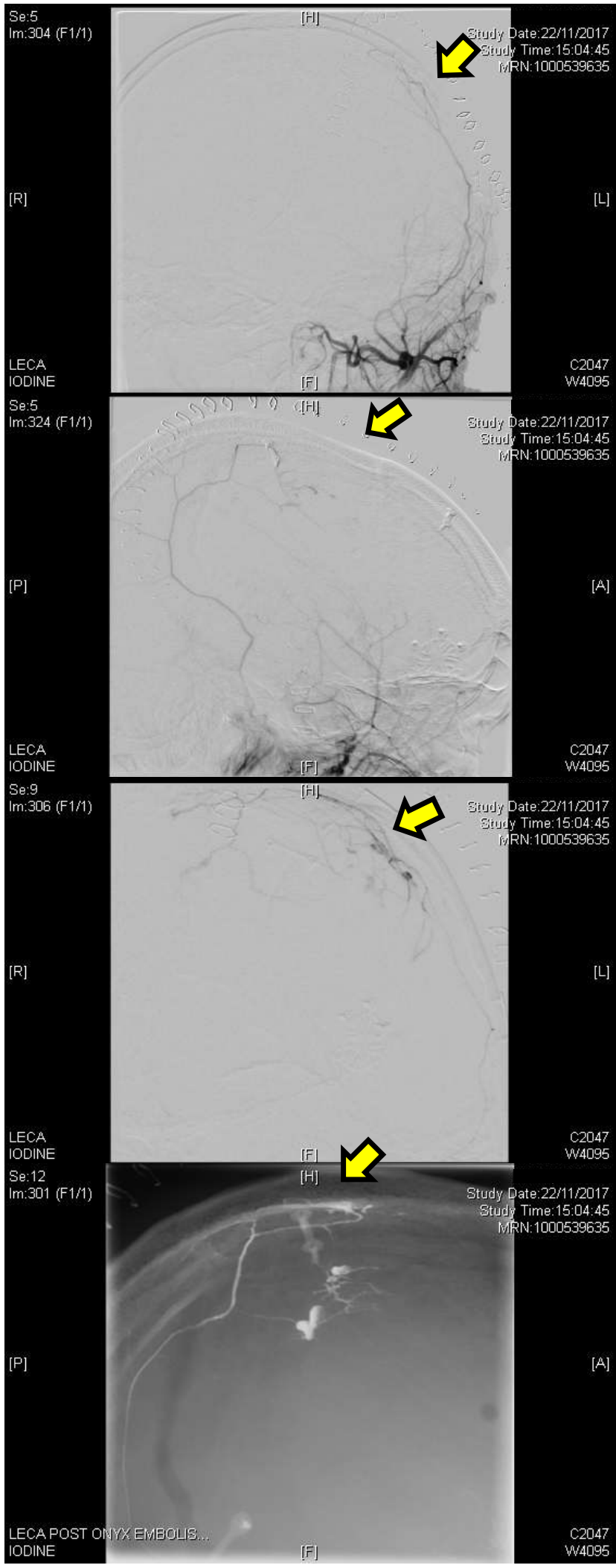

Figure 4. Digital Substraction Angiography Left External Carotid Artery (A, B). AP view dan B. Lateral view. It shows pseudoaneurysm of MMA. no vascular shunt or widening of drainage vein to sinus sagitalis superior that differentiate to dural AV malformation (DAVF) C. Catherization of superselective $L$ ECA shows of pseudoaneurysms of middle meningeal artery. D. Distal part of middle meningeal artery after filled with Onyx. It shows that onyx filled pseudoaneurysms. for 2 weeks because of lung embolism. This patients was undergone embolization of middle meningeal artery with Onyx and later craniotomy to evacuate the bleeding. ${ }^{5}$ Unlike this presented case, the age of the patients was old, with the risk factor of consuming anticoagulants drugs and the embolization was done before craniotomy.

Non traumatic acute spontaneous subdural hematoma was reported in young people of 27 year old male patient. This patient was reported had a dural arteriovena fistula (DAVF) or malformation and shunt artery to vein. Angiography was done before craniotomy and showed a shunt artery to vein, and widening of superior sagital sinus and sphenopalatine sinus as venous drainage. Embolization was performed with $25 \%$ diluted n-butyl cyanoacrylate (NBCA). ${ }^{6}$ Saito reported 9 cases of DAVF that caused spontaneous subdural hematoma. $^{7}$

Pseudoaneurysm or false aneurysm, is caused by a laseration in the arterial wall that produced local bleeding and turbulence of blood flow. Neck part of the pseudoaneuysm might not spontaneous closed when it reaches certain limits of size. It is unlikely the original aneurysm, it did not have arterial layer of wall like the real arterial wall. The wall is performed by the clot building process and it is consisted of platelets or fibrial bonding that weaker than actual aneurysm. It is also prone to rupture because the single layer of wall. That is the reason that this aneurysm type needs agressive treatment.

Only 28 cases of acute nontraumatic MMA aneurysms have been reported, many associated with high-flow condition, such as AVM, DAVF, Meningioma and moyamoya disease. The behaviour of these aneurysms is largely not well known. Some reported spontaneous resolution, others reported the growth of the aneurysm, leading to subsequent rupture. ${ }^{8}$ Marvin $^{9}$ reported that a pseudoaneurysm caused spontaneous subdural hematoma in 54 year old female patient with intraparenchymal bleeding in the right temporal. The occurence of pseudoaneurysm of middle meningeal artery is uncommon, the standard treatment are not well defined. ${ }^{9}$

The question why a pseudoaneurysm of middle meningeal artery manifestated as a subdural hematoma, instead of epidural hematoma might be answered by the number of layer of duramater. Duramater is consisted of three different layers. The outer layer is the thinnest with $2 \mu \mathrm{m}$ thick. The inner part is with $8 \mu \mathrm{m}$ thick and is attached to the trabeculae of arachnoid. The middle part is where the middle meningeal artery located is varied in its thickness. $^{9,10}$

Embolization of middle meningeal artery is a preventive treatment to avoid recurrencies in spontanous subdural hematoma chronic. The rate of recurrencies of subdural hematoma chronic is around $10 \%$. The inflammation and bleeding from outer membrane of subdural are caused the recurrencies. ${ }^{11}$ Superselective angiography of recurrency subdural hematoma patients show vascular blush along the arterial walls (macrocapillaries). Tempaku ${ }^{12}$ reported 5 cases of recurrencies of chronic sbdural hematoma and the results are satisfactory. Based on the outcome of embolization in chronic subdural hematoma that makes the embolization procedure in acute subdural hematoma had a good reason. 


\section{Conclusion}

Subdural Non traumatic spontaneous acute subdural hematoma is a rare entity and the source of bleeding of pseudoaneurysm of middle meningeal artery must be taken into account remembering the tendency of rebleeding. The diagnosis and agressive treatment are important, preventing devastating condition of their rupture. Embolization is one of the option to avoid the serious consequencie of recurrencies.

\section{Acknowledgment}

Greatest appreciation for Tim Herrington, Ken Faulder, Brandon Steinfort, neurointervensionists in Westmead Hospital, Sydney, Australia that have given supervision in writing this article.

\section{References}

1. Famaey N, Cui ZY, Musigazi GU, Ivens J, Depreitere B, Verbeken E, et al.: Structural and mechanical characterisation of bridging veins: A review. Journal of the mechanical behavior of biomedical materials. 2015;41:222-40. DOI: 10.1016/j.jmbbm.2014.06.009

2. Awaji K, Inokuchi R, Ikeda R, Haisa T: Nontraumatic Pure Acute Subdural Hematoma Caused by a Ruptured Cortical Middle Cerebral Artery Aneurysm: Case Report and Literature Review. J NMC case report journal. 2016;3(3):63-6.

DOI: $10.2176 /$ nmccrj.cr.2015-0151

3. Freire MA, Adriaensens MP, Marín AC. Spontaneous acute subdural hematoma. An unusual presentation of ruptured cerebral aneurysm. J Medicina intensiva; 2019.43(3):190-. DOI: 10.1016/j.medine.2019.01.003

4. Martins WA, Teixeira AB, Frigeri TM, Paglioli E: Spontaneous subdural hematoma associated to Duret hemorrhage. J Interdisciplinary neurosurgery; 2015. 2(1):13-5. DOI : 10.1016/j.inat.2014.11.003

5. Ramaiah G, Zaidi S, Jumaa M, Rao PM, Litzenberg K. Subdural hematoma secondary to spontaneous rupture of pseudoaneurysm of middle meningeal artery (P6. 234). AAN Enterprises; 2018. DOI:

6. Yamauchi K, Takenaka S, Iida T, Sakai H: A Case of Spontaneous Acute Subdural Hemorrhage Caused by a Dural Arteriovenous Fistula on the Convexity without Cortical Venous Reflux. J case reports in Neurology; 2019. 11(3):312-8.

DOI: $10.1159 / 000504290$

7. Saito A, Kawaguchi T, Sasaki T, Nishijima M. A case of dural arteriovenous fistula presenting as acute subdural hematoma. J case reports in Neurology; 2014. 6(1):122-5. DOI: 10.1159/000362116

8. Gerosa A, Fanti A, Del Sette B, Bianco A, Cossandi C, Crobeddu E, et al. Posttraumatic middle meningeal artery pseudoaneurysm: Case report and review of the literature. J world neurosurgery; 2019. 128:225-9. DOI: 10.1016/j.wneu.2019.05.030

9. Marvin E, Laws LH, Coppens JR: Ruptured pseudoaneurysm of the middle meningeal artery presenting with a temporal lobe hematoma and a contralateral subdural hematoma. J surgical neurology international; 2016. 7(Suppl 2):S23. DOI: $10.4103 / 2152-7806.173564$

10. Kohyama S, Kakehi Y, Yamane F, Ooigawa H, Kurita $\mathrm{H}$, Ishihara S. Subdural and intracerebral hemorrhage caused by spontaneous bleeding in the middle meningeal artery after coil embolization of a cerebral aneurysm. J Journal of Stroke Cerebrovascular Diseases; 2014. 23(9):e433-e5.

DOI: $10.1016 /$ j.jstrokecerebrovasdis.2014.05.01

11. Link TW, Boddu S, Paine SM, Kamel H, Knopman J. Middle meningeal artery embolization for chronic subdural hematoma: A series of 60 cases. J Neurosurgery; 2019. 85(6):801-7. DOI: DOI: $10.1093 /$ neuros/nyy521

12. Tempaku A, Yamauchi S, Ikeda H, Tsubota N, Furukawa H, Maeda D, et al. Usefulness of interventional embolization of the middle meningeal artery for recurrent chronic subdural hematoma: Five cases and a review of the literature. J Interventional Neuroradiology; 2015. 21(3):366-71. DOI: $10.1177 / 1591019915583224$ 